\title{
SOME STRUCTURAL REQUIREMENTS FOR THE PREVENTION OF LEUKOPENIA INDUCED BY NITROGEN MUSTARD ${ }^{1,2}$
}

\author{
By AUSTIN S. WEISBERGER, ROBERT W. HEINLE, AND BENNETT LEVINE \\ (From the Department of Medicine, Lakeside Hospital, and the School of Medicine, Western \\ Reserve University, Cleveland, Ohio)
}

(Submitted for publication September 19, 1951 ; accepted November 27, 1951)

Administration of L-cysteine hydrochloride to animals prior to the injection of nitrogen mustard $\left(\mathrm{HN}_{2}\right)$ modifies the severe leukopenia characteristically induced by $\mathrm{HN}_{2}$ (1). However, if the L-cysteine hydrochloride is administered after $\mathrm{HN}_{2}$, even immediately, the leukopenia is not modified. It has not been demonstrated whether the effect of L-cysteine depends upon the presence of certain groups or groupings in the molecule or whether it is the result of non-specific chemical inactivation of $\mathrm{HN}_{2}$. Accordingly, the specificity as well as the mechanism of L-cysteine protection was investigated by correlating the chemical structure of related compounds with their ability to prevent $\mathrm{HN}_{2}$-induced leukopenia.

\section{METHODS}

Several amino acids and sulfhydryl-containing compounds structurally closely related to cysteine, as well as compounds containing various combinations of functional sulfhydryl, amino and carboxyl groups were investigated. Ascorbic acid was also tested because, like the other compounds, it is a strong reducing agent.

In addition to L-cysteine, the compounds investigated were glutathione, D,L-homocysteine, D,L-methionine, L-alanine, D,L-serine, thioglycolic acid, dithiopropanol (BAL), thiomalic acid, thiourea, beta mercapto-ethylamine and ascorbic acid (Table I and Figures 1-4).

Except for dithiopropanol (BAL), amounts molecularly equivalent to, or greater than the amounts of $\mathrm{L}-\mathrm{Cy}-$ steine which have been found to be effective in preventing $\mathrm{HN}_{2}$-induced leukopenia in rabbits were used ( $\mathrm{T}$ able I). Larger amounts of BAL were not used because of their toxicity. Solutions of these substances were adjusted to pH 6.5 to 7.0 with sodium hydroxide and, with the exception of BAL, were injected either intravenously or intraperitoneally. Dithiopropanol (10 per cent solution of BAL in oil) was administered by intramuscular or intraperitoneal injection.

1 This study was made possible by a grant from the American Cancer Society, recommended by the Committee on Growth of the National Research Council.

2 Presented in part at the Central Society for Clinical Research, Chicago, November 2, 1951.
A freshly prepared saline solution of $\mathrm{HN}_{2}$ [methyl bis (beta chlorethyl) amine hydrochloride ${ }^{3}$ ] containing 1 mg. per $\mathrm{ml}$. was used in each instance. A single intravenous dose of $2.5 \mathrm{mg}$. of $\mathrm{HN}_{2}$ per $\mathrm{kg}$. of body weight uniformly induces a maximal leukopenia in rabbits in four days. The ability of the compounds studied to affect the leukopenia was determined by studying the daily variations in the total and differential leukocyte counts when

TABLE I

Prevention of leukopenia induced by nitrogen mustard

\begin{tabular}{|c|c|c|c|c|}
\hline \multirow{2}{*}{ Compound investigated } & \multicolumn{2}{|c|}{$\begin{array}{l}\text { Incubation with } \\
\text { nitrogen mustard } \\
\text { prior to injection }\end{array}$} & \multicolumn{2}{|c|}{$\begin{array}{l}\text { Compound } \\
\text { administered prior } \\
\text { to injection of } \\
\text { nitrogen mustard }\end{array}$} \\
\hline & $\begin{array}{l}\text { No. of } \\
\text { rabbits }\end{array}$ & $\begin{array}{c}\text { Maximum } \\
\text { fall in } \\
\text { WBCs } \\
\text { (\% of } \\
\text { control) }\end{array}$ & $\begin{array}{l}\text { No. of } \\
\text { rabbits }\end{array}$ & $\begin{array}{c}\text { Maximum } \\
\text { fall in } \\
\text { WBCs } \\
\text { (\% of } \\
\text { control) }\end{array}$ \\
\hline 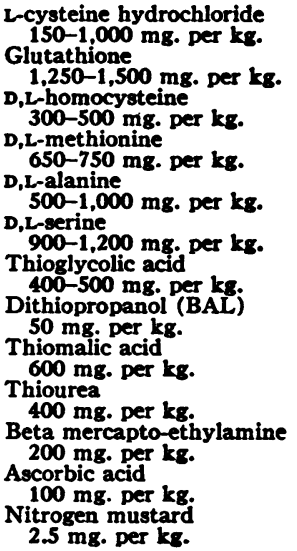 & $\begin{array}{l}7 \\
5 \\
- \\
5 * \\
5 \\
5 * \\
5 \\
5 * \\
3 \\
3 \\
- \\
6 \\
5 \ddagger\end{array}$ & $\begin{array}{c}16.6 \\
30.7 \\
- \\
0 \\
0 \\
23.0 \\
14.5 \\
26.6 \\
0 \\
36.6 \\
- \\
79.7 \\
85.3\end{array}$ & $\begin{array}{c}20 \\
6 \\
7 \\
5 * \\
12 \\
5 * \\
7 \\
8 \dagger \\
4 \\
5 \\
2 \\
5 \\
21\end{array}$ & $\begin{array}{l}37.5 \\
61.8 \\
65.3 \\
87.0 \\
80.8 \\
81.3 \\
90.3 \\
88.2 \\
79.2 \\
78.7 \\
77.7 \\
80.1 \\
79.9\end{array}$ \\
\hline
\end{tabular}

* Administered intraperitoneally.

$\uparrow$ Administered intramuscularly.

‡ Methyl bis (beta chlorethyl) amine hydrochloride incubated for 30 minutes at $37^{\circ} \mathrm{C}$.

these compounds were administered 10 to 15 minutes prior to the injection of $\mathrm{HN}_{2}$.

The effect of direct chemical interaction with $\mathrm{HN}_{2}$ was studied by incubating the compounds under investigation with $\mathrm{HN}_{2}$ in vitro at $37^{\circ} \mathrm{C}$. for 30 minutes in the same

3 The nitrogen mustard and L-cysteine hydrochloride used in these experiments were furnished through the courtesy of Merck \& Company, Rahway, New Jersey. 
<smiles>N[C@@H](CS)[C@H](O)C(=O)O</smiles>

CYSTEINE<smiles>C[C@H](C(=O)O)[C@@H](N)CS</smiles>

HOMOCYSTEINE

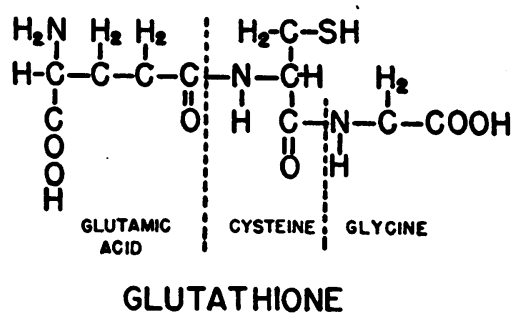

Fig. 1.
These compounds having vicinal sulfhydryl, amino and carboxyl groups are capable of modifying the leukopenia induced by nitrogen mustard. Substitution or alteration of either the free sulfhydryl, amino or carboxyl group results in the complete disappearance of any detectable protective effect against mustard-induced leukopenia.

amounts as were used in vivo. The resultant mixtures were then injected into rabbits and the changes in leukocyte count were determined. These changes were compared with the effect obtained when the same amounts of these compounds were given parenterally 10 to $15 \mathrm{~min}$ utes prior to the administration of the same amounts of $\mathrm{HN}_{\mathbf{2}}$.

\section{RESULTS}

With the exception of ascorbic acid, injection of incubated mixtures of $\mathrm{HN}_{2}$ and the various compounds caused very little leukopenia compared to<smiles>CCC(N)C(=O)O</smiles>

ALANINE<smiles>N[C@@H](CO)[C@H](O)C(=O)O</smiles>

SERINE<smiles>CS[C@@H]1CC1[C@H]([NH3+])C(=O)O</smiles>

\section{METHIONINE}

Fig. 2. Compounds Containing a Frer Amino and Vicinal Carboxyl Group

Although these compounds are structurally closely related to cysteine, they lack a free sulfhydryl group and exert no protective effect against mustard-induced leukopenia. that which would have been produced by the same amount of $\mathrm{HN}_{2}$ alone. The maximum decrease in the leukocyte count following the injection of a saline solution of $\mathrm{HN}_{2}$ which had been incubated by itself for 30 minutes at $37^{\circ} \mathrm{C}$. was 85.3 per cent of the control count. Administration of the same dose of $\mathrm{HN}_{2}$ following incubation with equivalent amounts of L-cysteine, glutathione, D,L-methionine, D,L-alanine, D,L-serine, thioglycolic acid, $\mathrm{BAL}$, thiomalic acid, and thiourea resulted in a decrease in the leukocyte count ranging from 0 to 36.6 per cent of the control counts (Table I, Figure 5). The differential leukocyte count did not change. There was no significant decrease in the degree of leukopenia induced by $\mathrm{HN}_{2}$ following

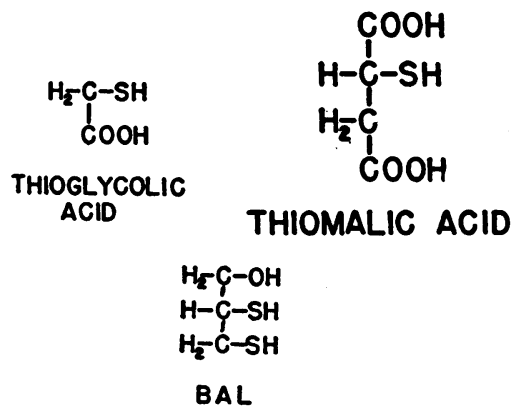

Fig. 3. Compounds Containing Free Sulfhydryl GROUPS

Although some of these compounds contain a carboxyl group in addition to the sulfhydryl group, they do not contain an amino group. These compounds fail to prevent the mustard-induced leukopenia. The presence of two free sulfhydryl groups in BAL does not result in protection against the leukopenia induced by nitrogen mustard.

its incubation with ascorbic acid. Chemical interaction between $\mathrm{HN}_{2}$ and most of the compounds studied did not occur at room temperature, but only after incubation at $37^{\circ} \mathrm{C}$. for 30 minutes.

In contrast to the results obtained after administration of the incubated mixtures were those obtained when equivalent amounts of the different compounds were injected prior to the $\mathrm{HN}_{2}$. Only L-cysteine and to a much lesser extent D,L-homocysteine and glutathione were capable of modifying the leukopenia (Table I, Figure 6). The average maximum fall in the leukocyte count induced by $\mathrm{HN}_{2}$ was 79.9 per cent of the control counts. When L-cysteine was injected prior to $\mathrm{HN}_{2}$, the average maximum fall in the leukocyte 
count was 37.5 per cent of the control counts. Injection of equivalent amounts of glutathione and D,L-homocysteine prior to $\mathrm{HN}_{2}$ resulted in an average maximum fall in the leukocyte count of 61.8 and 65.3 per cent of the control counts, respectively.

Injection of equivalent amounts of the other compounds prior to the injection of $\mathrm{HN}_{2}$ resulted in a maximum fall in the leukocyte count ranging from 78.7 to 90.3 per cent of the control counts, compared with the fall of 79.9 per cent with $\mathrm{HN}_{2}$ alone. The most severe leukopenia occurred when either thioglycolic acid, BAL, or methionine was injected prior to $\mathrm{HN}_{2}$ (Table I). The maximum leukopenia invariably occurred on the fourth or fifth day following injection.

$$
\begin{aligned}
& \mathrm{H}_{2}-\mathrm{C}-\mathrm{SH} \\
& \mathrm{H}_{2}-\mathrm{C}-\mathrm{NH}_{2}
\end{aligned}
$$

\section{BETA MERCAPTO ETHYLAMINE}

Fig. 4. Compound Containing a Free Sulfhydryl and Amino Group without a Carboxyl Group

Although this compound has the same structural configuration as cysteine except for the absence of a carboxyl group, it does not prevent mustard-induced leukopenia.

Injection of the compounds without subsequent administration of $\mathrm{HN}_{2}$ did not cause a significant change in the leukocyte counts.

\section{DISCUSSION}

It is apparent that many highly reactive compounds with structures closely related to cysteine do not modify the leukopenia induced by $\mathrm{HN}_{2}$. The modification of mustard-induced leukopenia appears to depend upon the presence of certain specific groups in close apposition in the molecule. An analysis of some of the structural requirements reveals the following:

1. The role of vicinal sulfhydryl, amino and carboxyl groups: A comparison of the structure of the compounds studied with the ability to pre-



Fig. 5. Average Leukocyte Counts Obtained When Nitrogen MUSTaRd Is INCUbated IN VITRO With Various Compounds Prior to Injection

L-Cysteine, glutathione, methionine, alanine, serine, thioglycolic acid, BAL, thiomalic acid and thiourea inactivate mustard when allowed to react directly with $\mathrm{HN}_{2}$ prior to administration. However, only L-cysteine, glutathione and D,L-homocysteine modify mustard-induced leukopenia when injected in vivo.

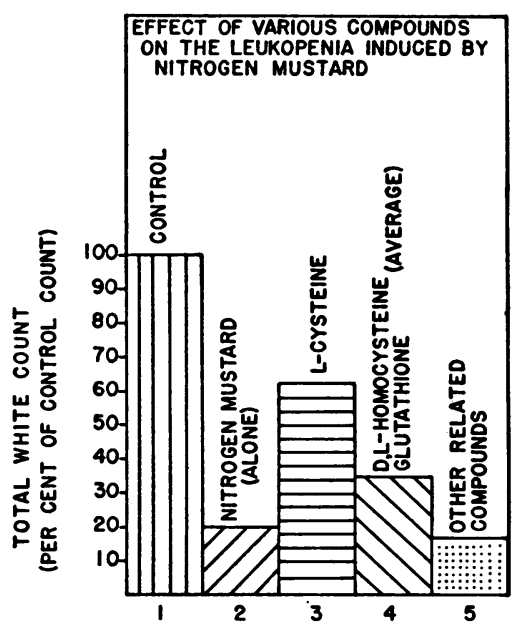

Fig. 6. Average Leukocyte Counts Obtained When Nitrogen Mustard is Injected after the AdministraTION OF VARIOUS COMPOUNDS

L-Cysteine is the most effective in preventing leukopenia (column 3). D,L-homocysteine and glutathione are not as effective (column 4). Methionine, alanine, serine, thiomalic acid, thioglycolic acid, BAL, thiourea and ascorbic acid are completely ineffective in modifying the mustard-induced leukopenia (column 5). 
vent leukopenia reveals that only those compounds which have a sulfhydryl, amino and carboxyl group in close apposition were capable of preventing $\mathrm{HN}_{2}$-induced leukopenia (Figure 1). Thus, L-cysteine, D,L-homocysteine and glutathione had a definite protective effect. L-Cysteine, which has a carboxyl group, a free sulfhydryl group, and an amino group on adjacent carbon atoms, was by far the most effective. D,L-Homocysteine, in which the free sulfhydryl and free amino group are separated by one carbon atom, was much less effective than L-cysteine. It is possible that one of the optical isomers of homocysteine may not be effective in preventing leukopenia and that the decreased protection was due to the presence of an inactive isomer in the $D, L$ mixture of the amino acid. Methylation of the sulfhydryl group in D,L-homocysteine to form D,Lmethionine (Figure 2) resulted in the complete disappearance of any detectable protective effect.

Glutathione is composed of one molecule of glutamic acid, one of cysteine, and one of glycine (Figure 1). The partial prevention of $\mathrm{HN}_{2}$-induced leukopenia by glutathione may be attributed to the presence of cysteine in the molecule. Although the amount of glutathione injected contained sufficient cysteine (as a constituent of glutathione) to furnish satisfactory protection, the prevention of leukopenia was much less than that obtained with equivalent amounts of L-cysteine. This may indicate that the conjugation of the amino or carboxyl group, or both, in cysteine to form glutathione resulted in decreased ability to protect against leukopenia, or that the release of cysteine from glutathione in vivo was too slow to furnish optimum protection.

2. The effect of compounds containing vicinal amino and carboxyl groups without an adjacent sulfhydryl group: The injection of compounds containing a free amino and vicinal carboxyl group without a sulfhydryl group resulted in the absence of any protective effect. Thus, the replacement of the sulfhydryl group in cysteine with an hydroxyl group to form serine or with hydrogen to form alanine (Figure 2) resulted in complete failure of protection. Thiourea $\left(\mathrm{NH}_{2} \cdot \mathrm{CS} \cdot \mathrm{NH}_{2}\right)$, containing two amino groups without vicinal carboxyl or sulfhydryl groups, was likewise ineffective.
3. The role of free sulfhydryl groups: Compounds containing a free sulfhydryl group without an amino group in close proximity were ineffective also, even though one or two carboxyl groups were adjacent (thioglycolic and thiomalic acid, Figure 3 ). The failure of BAL, which has two vicinal sulfhydryl groups, to manifest a protective effect is further evidence that the presence in itself of sulfhydryl groups is not sufficient to prevent leukopenia.

4. The role of the carboxyl group: The presence of a carboxyl group in addition to vicinal amino and sulfhydryl groups is essential for a protective effect. Thus beta mercaptoethylamine which has the same structural configuration as cysteine without a carboxyl group (Figure 4) does not modify $\mathrm{HN}_{2}$-induced leukopenia. This compound was very toxic, several animals dying within three to four hours following intravenous or intraperitoneal administration. The cause of death in these animals is unknown. No irritation or adverse reaction was noted during the period of injection nor immediately after.

Thus under the conditions of these experiments, neither the reducing potential of a compound, nor the presence by themselves of free carboxyl, sulfhydryl, or amino groups could be correlated with the ability of the compounds studied to prevent leukopenia when injected prior to $\mathrm{HN}_{2}$. Those compounds exhibiting a protective effect uniformly have in common the presence of a closely apposed free sulfhydryl, amino and carboxyl group.

The mechanism by which this specific structural configuration results in modification of the $\mathrm{HN}_{2}$-induced leukopenia is not known. The lack of sparing effect of closely related compounds could conceivably indicate only that the affinity of these compounds for $\mathrm{HN}_{2}$ in vivo is not as great as that of L-cysteine, D,L-homocysteine and glutathione; even though they are able to combine with $\mathrm{HN}_{2}$ in vitro. It is possible that the presence of vicinal sulfhydryl, amino and carboxyl groups permit intracellular penetration thereby enabling these compounds to inactivate $\mathrm{HN}_{2}$. However, L-cysteine likewise modified the leukopenia induced by irradiation (2) where chemical inactivation of

\footnotetext{
- The beta mercaptoethylamine used in these experiments was prepared and furnished through the courtesy of Dr. Sam Seifter, State University of New York, College of Medicine at New York City.
} 
noxious substances is presumably not involved. It is possible, therefore, that L-cysteine protection of leukocytes against damage by $\mathrm{HN}_{2}$, as with irradiation, is not due solely to chemical inactivation.

Fruton and associates $(3,4)$ have shown that $\mathrm{HN}_{2}$ combines in vitro with the functional groups of many proteins and important biologic substances. They suggested that $\mathrm{HN}_{2}$ may produce its effects by inactivating similar substances in vivo. It may then be postulated that $\mathrm{HN}_{2}$-induced leukopenia results from the inactivation or destruction of some of these substance(s) by $\mathrm{HN}_{2}$ which are essential for normal leukopoiesis, and that L-cysteine or related compounds exert a sparing or protective effect by virtue of competition with $\mathrm{HN}_{2}$.

There is abundant evidence that sulfhydryl-containing compounds are involved in leukopoiesis. It is known, for example, that sulfhydryl compounds are concerned with cell division and growth, that the sulfhydryl content of immature cells is greater than that of mature cells and that the blood sulfhydryl content is low in hypoplastic states (5). Furthermore, irradiation and drugs that produce a granulocytopenia also produce a fall in the blood sulfhydryl level. A specific molecular configuration involving sulfhydryl, amino and carboxyl groups may be involved in these phenomena. Thus L-cysteine and related compounds may have a role in leukocyte formation or in abnormal leukopoiesis. Further investigation of the role of cysteine and cysteine analogues in leukocyte metabolism is in progress.

The effect of D-cysteine as compared with L-cysteine was not investigated because of the unavailability of D-cysteine. It has been shown (6), however, that L-cysteine can reverse cysteine deficiency produced in rats fed low cysteine diets containing bromobenzene, whereas D-cysteine is ineffective. If $\mathrm{D}$-cysteine were similarly ineffective in preventing the leukopenia induced by $\mathrm{HN}_{2}$, it would be further evidence that the mechanism of the L-cysteine protection is other than that solely of chemical inactivation of $\mathrm{HN}_{2}$ in vivo.

The failure of $\mathrm{HN}_{2}$ to produce leukopenia when injected after incubation in vitro with most of the compounds studied, indicates that the $\mathrm{HN}_{2}$ was inactivated by this process. $\mathrm{HN}_{2}$ undergoes rapid intramolecular rearrangement in aqueous solution to form an imine linkage (7). In this form it is highly reactive and is capable of alkylating free sulfhydryl, amino and carboxyl groups (3). It is likely that alkylation of these groups in the compounds studied was permitted to take place during direct chemical interaction in vitro, thereby resulting in loss of potency of the $\mathrm{HN}_{2}$. While the reaction efficiency of these compounds was not determined, the protective effect of L-cysteine against leukopenia induced by irradiation indicates that factors such as blood level and solubility may not be involved in the failure of compounds with closely related structure to prevent leukopenia.

\section{SUMMARY AND CONCLUSIONS}

1. The specificity and mechanism of L-cysteine protection against $\mathrm{HN}_{2}$-induced leukopenia were investigated by studying the relationship of structure to ability to prevent leukopenia.

2. Of the compounds studied, only those with vicinal sulfhydryl, amino, and carboxyl groups were capable of modifying $\mathrm{HN}_{2}$-induced leukopenia. L-Cysteine, which has a free sulfhydryl, amino and carboxyl group on adjacent carbon atoms was the most effective in preventing leukopenia. D,L-Homocysteine and glutathione were less effective.

3. Substitution or alteration of either the free sulfhydryl, amino or carboxyl group results in the complete disappearance of any detectable protective effect against $\mathrm{HN}_{2}$-induced leukopenia.

4. Under the conditions of this experiment, neither the reducing action of a compound, nor the presence by themselves of free sulfhydryl, amino or carboxyl groups can be correlated with the ability of the compounds studied to prevent leukopenia when injected prior to nitrogen mustard.

5. The data indicate that the protective effect of L-cysteine and its homologues in vivo does not result solely from inactivation of $\mathrm{HN}_{2}$. It is more likely that L-cysteine protects some substance(s), essential for leukopoiesis, from destruction by $\mathrm{HN}_{2}$.

\section{REFERENCES}

1. Weisberger, A. S., and Heinle, R. W., The protective effect of cysteine on leukopenia induced by nitrogen mustard. J. Lab. \& Clin. Med., 1950, 36, 872. 
2. Rosenthal, R. L., Goldschmidt, L., and Pickering, B. I., Hematologic changes in rats protected by cysteine against total body X-irradiation. Am. J. Physiol., 1951, 166, 15.

3. Fruton, J. S., Stein, W. H., and Bergmann, M., Chemical reactions of the nitrogen mustard gases. V. The reactions of the nitrogen mustard gases with protein constituents. J. Organic Chem., 1946, 11, 559.

4. Fruton, J. S., Stein, W. H., Stahmann, M. A., and Golumbic, C., Chemical reactions of the nitrogen mustard gases. VI. The reactions of the nitrogen mustard gases with chemical compounds of biological interest. J. Organic Chem., 1946, 11, 571.

5. Contopoulos, A. N., and Hamilton, H. H., Sulfhydryl content of blood cells in dyscrasias. J. Lab. \& Clin. Med., 1950, 36, 929.

6. Stekol, J. A., Studies on mercapturic acid synthesis in animals. XV. On the mechanism of inhibition of growth of rats by benzyl chloride. J. Biol. Chem., 1947, 167, 637.

7. Golumbic, C., Fruton, J. S., and Bergmann, M., Chemical reactions of the nitrogen mustard gases. I. The transformations of methylbis ( $\beta$ chlorethyl) amine in water. J. Organic Chem., 1946, 11, 518. 